\title{
Applied TRIZ in Improving Productivity in Textile Industry
}

\author{
Aminah Ahmad ${ }^{1, a}$, Nor Hamizah Miswan ${ }^{1}$, Rahaini Mohd Said ${ }^{1}$ and Rohana Abdullah ${ }^{1}$ \\ ${ }^{1}$ Universiti Teknikal Malaysia Melaka, UTeM, 71600, Durian Tunggal, Melaka, Malaysia
}

\begin{abstract}
TRIZ is a methodology and a collection of problem solving tools and strategies that has been used in many other fields. Therefore, this paper proposes TRIZ method for improving the productivity in a textile industry. It focuses at the packing department in a textile company situated in Malaysia. The process was monitored and the problem was observed. TRIZ method is applied in this problem using Functional Analysis and trimming method. A comparison between before and after implementation is done in order to evaluate the productivity effectiveness.
\end{abstract}

\section{Introduction}

Profitability of a firm much relies on productivity improvements. Furthermore, it is a common knowledge that the productivity improvement is one of the most important ways to increase competitiveness and especially in price competitiveness. Proper planning on productivity is possible to reduce the unit's costs of products and services.

The study is performed at a textile industry where productivity evaluation initiatives had been carried out for the purpose of increasing current productivity. The study will focus at the packaging department since it is one of the important areas in the company. There are two key aspects are considered in this department; packaging and labeling process.

Most of the packaging processes at the packaging area have a weighing step. Two types of weighing equipment are used, one is manual weight and the other one is digital weight. The main concern in this study is to define which one is more efficient to be used and more accurate. During the load process the operators load the items manually into the plastic bag and into a dritz box. This manual step is seen as waste because it is time consuming and causes the operators to easily get tired.

The entire dritz box used in packing needed a label at bottom of the box. This step again is done manually by pulling out the label from a piece of paper and then sticking the label to the dritz box. The label sticker material is made of plastic and it is difficult for the operators to separate the plastic label from the paper. Therefore, the operator had to use their nail to pull out the label and it is also another time consuming task.

\section{The method of finding and solving the problem using functional analysis.}

Functional Analysis is one of the concepts in TRIZ, which assist in building a comprehensive model of the Engineering System. Functional analysis consists of three steps, which are Component Analysis, Interaction Analysis and Function Model. The components analysis identifies components of the Engineering system and its supersystem, whereas interaction analysis identifies the interaction between components. Then, function model identifies and evaluate the functions performed by the components. Figure 1 shows the function model for this problem.

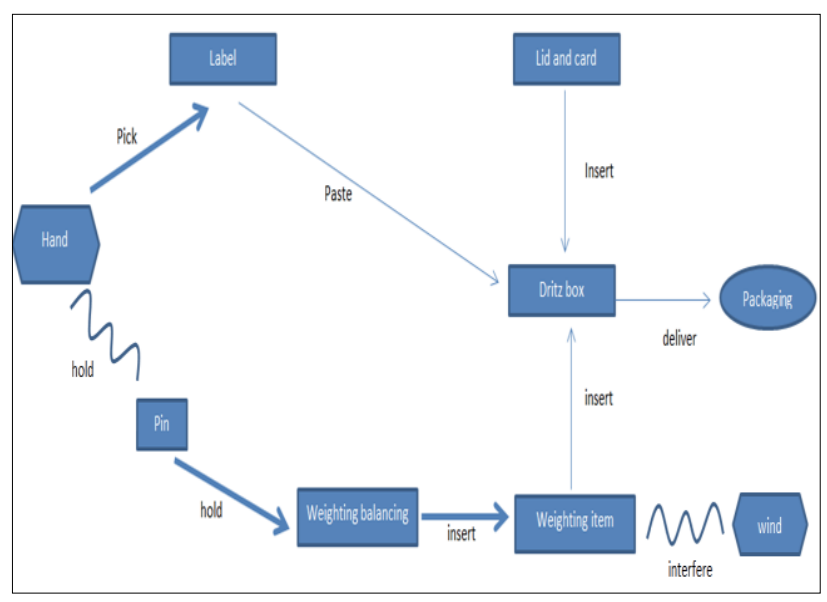

Figure 1. Functional Analysis before implementation.

\footnotetext{
a Corresponding author: aminah@utem.edu.my
} 
From Figure 1, the process starts with the operator's hand holding the pin. The interaction between hand and pin are harmful since the operator might injure their hands while performing the process. Next, the operator needs to weigh and balance the pins to ensure the weight meets the required weight specification before inserting the pin into a dritz box. During the weighing process, harmful supersystem such as wind is identified since it can affect the measurement accuracy.

Next, the dritz box will need to be labeled using a sticker. The labeling process is an exhaustive process where it requires high focus because the label needs to be put at the specific position at the bottom of the box and therefore, proper adjustment needed to be made before the label can be attached. This step is done manually by separating the plastic label from the paper, observing, adjusting the position of the sticker and finally sticking the label to the dritz box. Separating the label from the paper is very tedious and time consuming since the operator will need to use their nails to pull out the label from the paper. Finally, once the weighting and labeling process are done, a card is inserted and the dritz box's lid is closed and the part is ready to be delivered to the packaging department.

\section{Results and Discussion.}

Based on the analysis done, several improvements are proposed. The Functional Analysis results proposed is shown in Figure 2 below.

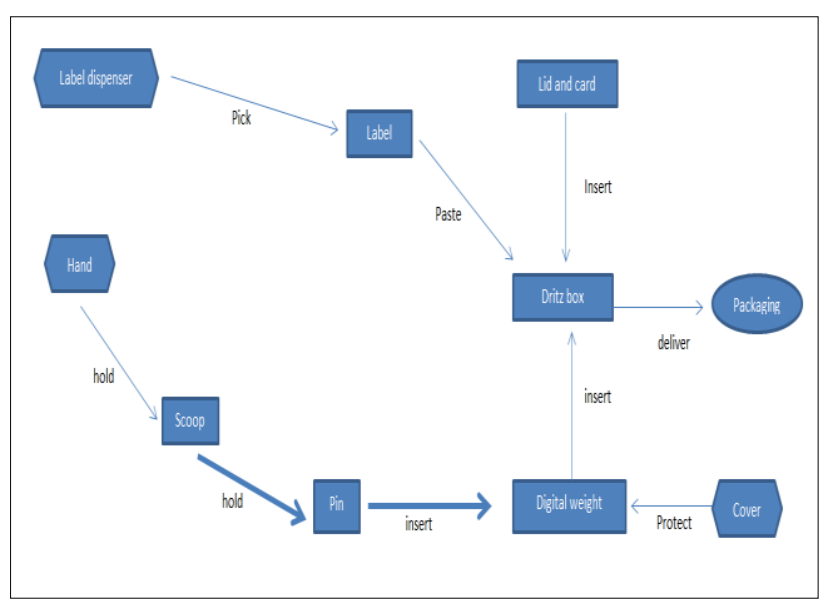

Figure 2. Functional Analysis after implementation.

Based on Figure 2, some improvements have been suggested at three process including weighting process, load bulk pack and stick label to dritz box by using trimming process in TRIZ.

\subsection{Improvement At Weighting Process}

The improvement that is suggested in this process is to change all the weighing equipment from balancing weight to digital weight. Figure 3 shows the balancing weight that has been used before improvement. Figure 4 shows the improvement of weighing process by using digital weight. The new time taken to weigh using digital weight is compared with the previous method. The results showed that using digital weight reduces the time taken to perform the weighing activity. In addition, kaizen improvement is also applied in this step where a cover is located around the digital weight to prevent the wind from affecting the reading of the weight and to ensure more accurate reading.

In Figure 5, the histogram shows the comparison between before and after improvement in term of average time. There is an improvement where the average time is reduced from 6.99 seconds to 5.92 seconds. It is about $16 \%$ improvement from the previous method.

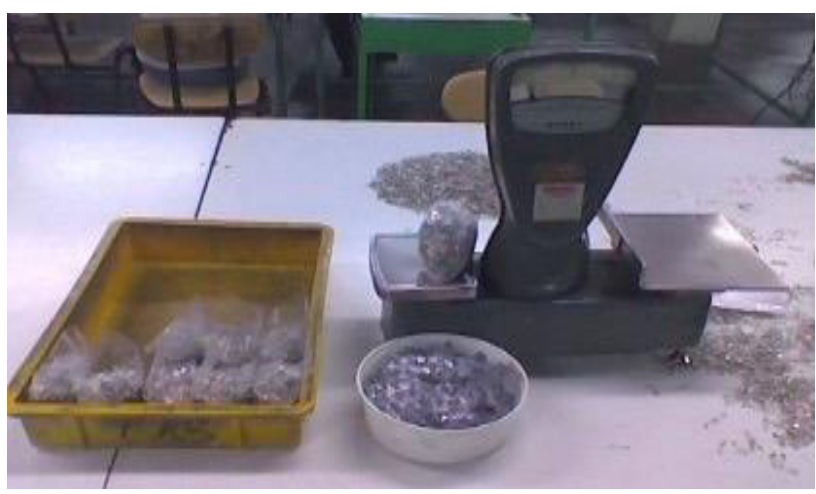

Figure 3. Before Improvement.

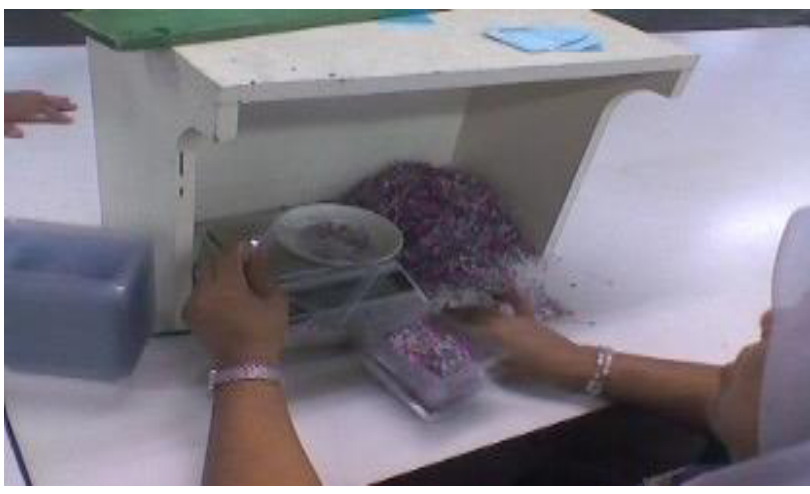

Figure 4. After Improvement.

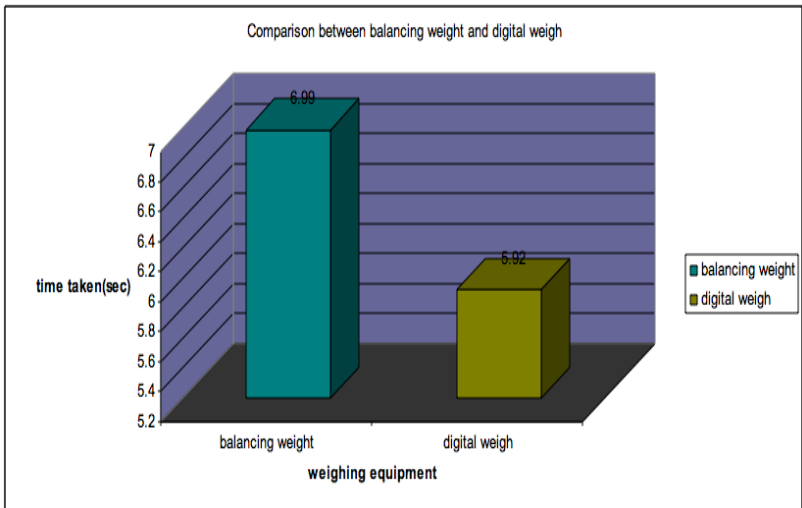

Figure 5. Histogram for comparison between before and after improvement at the weighing method. 


\subsection{Improvement At Load Bulk Pack}

As mentioned earlier and is shown in Figure 4, the weighing activity is done manually where it requires the operator to pick the pins using their bare fingers and this will cause the pins to prick and injure the fingers. The activity of picking, estimating, weighing and loading the pins is termed as the load bulk pack process. In this process, the improvement that is suggested is loading the item into pollybag using a scoop. The prototype of the scoop is designed and made using the stainless steel. From the observation, it is more efficient compared to the manual load since it's safer for the fingers and faster because the capacity of the scoop is already designed to meet the packaging weight. This means when using the scoop, it is much easier for the operator to estimate the item's weight requirement and lesser time is spent on this operation. Figure 8 shows the difference between previous method and the new methods. The average time is reduced from 23.24 seconds to only 9.03 seconds ( $62 \%$ improvement). This shows that using the measurement scoop is a much more efficient and safer method than using the bare fingers.

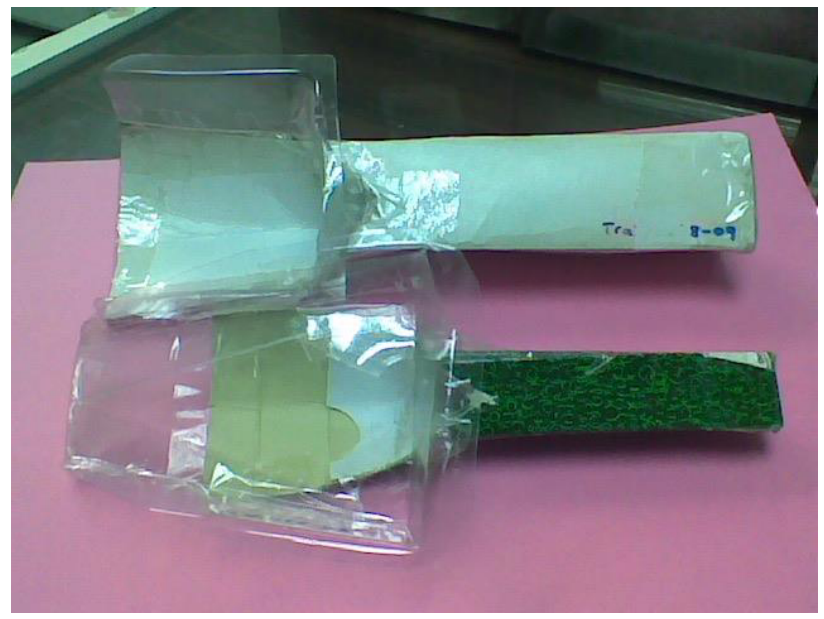

Figure 6. Scoop Prototype using hard cardboard.

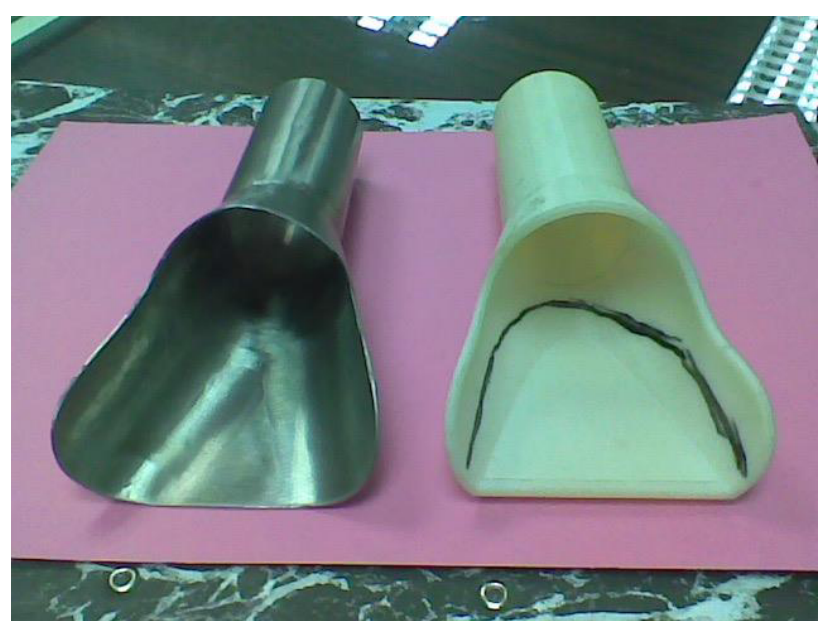

Figure 7. Scoop Prototype using stainless steel and polymer.

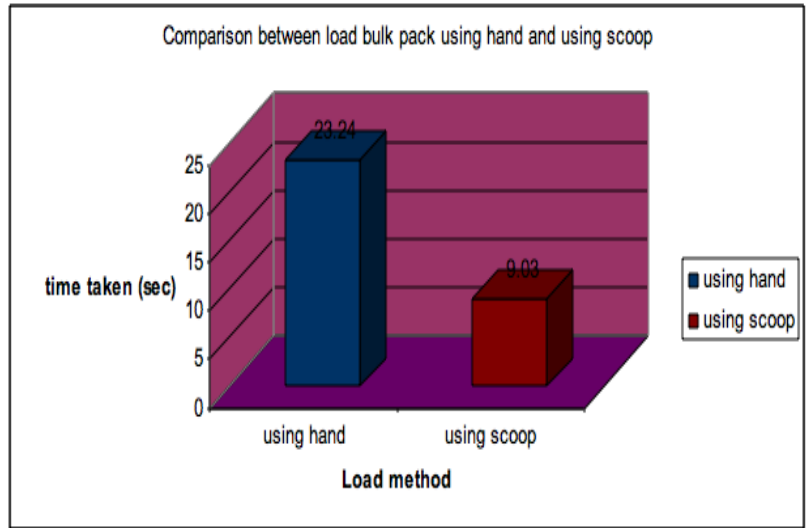

Figure 8. Histogram of comparison between load bulk pack using hand and using scoop.

\subsection{Improvement At Load Bulk Pack}

In this process, the main concern is to separate the plastic label from the paper and stick to the dritz box. The improvement suggested is to use a dispenser to remove the label from the paper instead of using the nails. This suggestion is made after reviewing several case studies. Many companies have implemented using the electric dispenser to replace the manual activity. However, for this study, a simple manual sticker dispenser was designed and fabricated based on the case studies' design of dispensers. The dispenser is designed using solid work software. The prototype is built using stainless steel material. From the time study, by using this dispenser, the average time is reduced from 3.23 seconds to 2.61 seconds $(20 \%$ improvement). Figure 10 shows the improvement of the stick label activity between using the nails and using the dispenser.

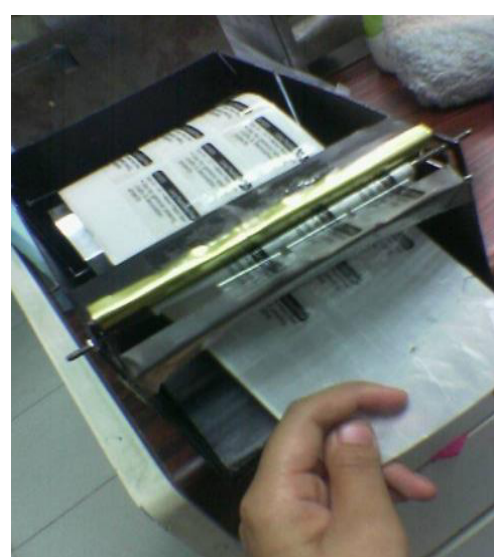

Figure 9. The label/sticker Dispenser Usage 


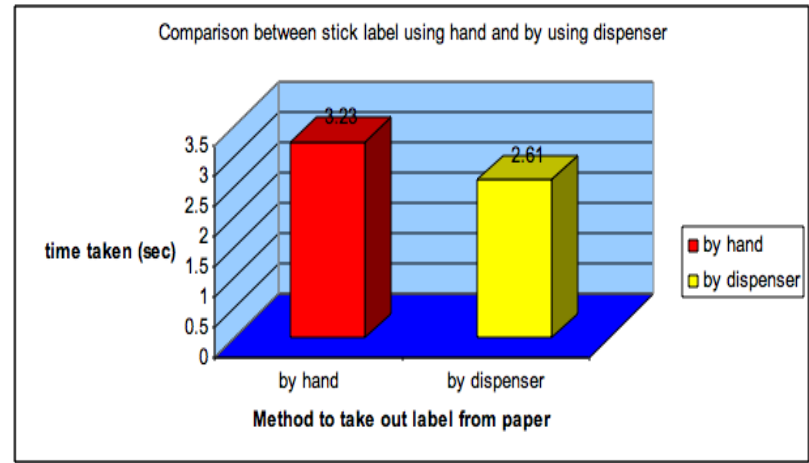

Figure 10. Histogram of comparison between before and after improvement of stick label method

\section{Conclusions}

The aim to study and improve the efficiency of the packaging department process at the textile company is achieved. The Functional Analysis approach in TRIZ is proven to be a very effective method to solve a problem where a problem is first analyzed using a functional analysis model and then the trimming process is done in order to obtain the improved Functional Analysis. After improvement activities has been implemented, comparisons in terms of the time taken between the before and after are done in order to measure the effectiveness of the productivity between before and after improvement.

\section{References}

1. Yeoh, T.S.,Yeoh, T.J and Song, C.L, TRIZ : Systematic Innovation in Manufacturing. Malaysia: Firstfruits, (2009).

2. D.Daniel Sheu and Chuan Ting Hou, TRIZ-based problem solving for process-machine improvements: Slit-valve Innovative redesign, Proceeding of the 41st International Conference on Computer \& Industrial Engineering (2013).

3. Valery Krasnoslobodtsev and Richard Langevin, Applied TRIZ in High Tech Industry, The TRIZ Journal, (2006).

4. Zhang Ruihong, Tan Runhua, and Cao Guazhong, Case study in ad and TRIZ: A paper Machine, The TRIZ Journal, (2004).

5. Nuralesya Ramlee, Productivity Improvement in Textile Industry. Malaysia: UTeM library, (2010).

6. Meyers F. E. and Stephens M. P., Manufacturing Facilities Design and Material Handling 3rd Ed. New Jersey: Prentice Hall, (2005).

7. Amrine H. T., Ritchey J. A., Moodie C. L. and Kmec J. F., Manufacturing Organization and Management 6th Ed. New Jersey: Prentice Hall.(1993)

8. Longenecker, C.O. and Stansfield, T.C., Why plant managers fail: causes and consequences, Industrial Management, (2000).

9. Stevenson J. W., Operations Management. New York: Mc Graw Hill(2007).
10. Ali M.M, Implementation of the DMAIC Analytical Method on Industrial Machinery Repair Service Company in Indonesia, (2008) 